\title{
Safety and efficacy of exposure to bedaquiline-delamanid in multidrug- resistant tuberculosis: a case series from France and Latvia
}

\author{
To the Editor:
}

Multidrug-resistant (MDR) and extensively drug-resistant (XDR) tuberculosis (TB) represent a therapeutic challenge [1]. Two anti-TB agents, bedaquiline and delamanid, have been recently approved for the treatment of MDR/XDR-TB. Bedaquiline has a terminal half-life of 5.5 months, in contrast with the short half-life of delamanid $(38 \mathrm{~h}$ ). Both drugs increase the QTcF (Fridericia-corrected QT) interval, although no clinically significant cardiac events have been reported in patients treated with one of these drugs $[2,3]$. The clinical trials that led to approval of bedaquiline and delamanid tested these drugs by adding one of them, for a duration of 24 weeks and 6-8 months, respectively, to optimised background [2, 3]. To date, only six cases treated with the bedaquiline-delamanid combination have been reported, mostly presenting interim results [4-6]. The World Health Organization (WHO) recommends to use these drugs for a standardised duration of 24 weeks, the concomitant use of bedaquiline and delamanid being restricted to patients with "no other therapeutic options". Otherwise, it is recommended to start the two drugs sequentially after a washout period of 6 months, when switching from bedaquiline to delamanid, and 5 days, when switching from delamanid to bedaquiline [7]. We report the results of exposure to concomitant and sequential treatment with bedaquiline and delamanid, including treatment courses beyond 24 weeks, as part of multidrug MDR/XDR-TB regimens.

A multicentre case series was established including patients who started treatment for culture-proven MDR-TB from January 1, 2013 to August 31, 2015, and were exposed to the bedaquiline-delamanid combination for $\geqslant 30$ days. Participating centres were three hospitals in the Paris region, France (Bligny, Pitié-Salpêtrière and Bichat), and the Riga East University Hospital in Riga, Latvia. Exposure to bedaquiline and delamanid was defined as the sum of: 1) days of concomitant treatment; and 2) days of exposure to one drug after stopping it, as defined by washout period, while taking the other one (sequential treatment). Standard definitions of MDR/XDR-TB and WHO treatment outcomes were applied. Time to sputum culture conversion was measured from treatment start to the first of two consecutive negative sputum culture results. Treatment regimens were tailored for each patient in agreement with WHO guidance: concomitant or sequential treatment with bedaquiline and delamanid was used to provide regimens with at least four effective drugs to patients with resistance or intolerance to second-line drugs. Bedaquiline and delamanid treatment courses were prolonged beyond 24 weeks according to criteria presented elsewhere [8]. Individualised treatment oversight was provided by the French and Latvian TB Consilia. Adequate treatment monitoring, including periodic ECGs as recommended, and pharmacovigilance were implemented. Treatment was usually started under inpatient, and then continued under outpatient care. Seriousness of adverse events was defined according to the US National Institutes of Health CTCAE version 4.0 [9]. Data were collected retrospectively from medical records. Ethics approval for the study was granted by the institutional review board of the coordinating centre in France (Bligny Hospital), and of the University of Latvia. Patients were informed about potential benefits and risks of treatment, including bedaquiline-delamanid exposure, and provided verbal consent to undergo the treatment.

@ERSpublications

Good tolerability of exposure to bedaquiline-delamanid association in MDR/XDR-TB patients http://ow.ly/4uvr30i7az4

Cite this article as: Guglielmetti L, Barkane L, Le Dû D, et al. Safety and efficacy of exposure to bedaquiline-delamanid in multidrug-resistant tuberculosis: a case series from France and Latvia. Eur Respir J 2018; 51: 1702550 [https://doi.org/10.1183/13993003.02550-2017]. 
TABLE 1 Characteristics of the 10 multidrug-resistant pulmonary tuberculosis patients exposed to bedaquiline and delamanid treatment

\begin{tabular}{|c|c|c|c|c|c|c|c|c|c|c|c|c|c|c|}
\hline Patient & $\begin{array}{l}\text { Age } \\
\text { years }\end{array}$ & TB form & $\begin{array}{c}\text { Resistance profile } \\
\text { (in addition to } \mathrm{H} \\
\text { and } R \text { ) }\end{array}$ & $\begin{array}{l}\text { Bdq-Dlm } \\
\text { treatment }^{\#}\end{array}$ & $\begin{array}{l}\text { Treatment } \\
\text { duration } \\
\text { with Bdq/ } \\
\text { Dlm days }\end{array}$ & $\begin{array}{l}\text { Total } \\
\text { treatment } \\
\text { duration } \\
\text { days }\end{array}$ & $\begin{array}{l}\text { Exposure } \\
\text { to Bdq and } \\
\text { Dlm days" }\end{array}$ & $\begin{array}{l}\text { Treatment: } \\
\text { non- } \\
\text { QT-prolonging } \\
\text { drugs }\end{array}$ & $\begin{array}{l}\text { Treatment: } \\
\text { QT-prolonging } \\
\text { drugs }\end{array}$ & $\begin{array}{l}\text { Maximum } \\
\text { QTcF value } \\
\text { ms } \\
\text { (months of } \\
\text { treatment) }\end{array}$ & $\begin{array}{c}\text { Drug } \\
\text { discontinued for } \\
\text { any adverse } \\
\text { event (adverse } \\
\text { event) }\end{array}$ & Surgery & $\begin{array}{l}\text { Time to } \\
\text { sputum } \\
\text { culture } \\
\text { conversion }\end{array}$ & $\begin{array}{l}\text { Treatment } \\
\text { outcome }\end{array}$ \\
\hline 1 & 55 & $\begin{array}{c}\mathrm{S}+\mathrm{C}+ \\
\mathrm{Cav}+\mathrm{Bil}+\end{array}$ & Z, E, Ofx, Eto, PAS & Concomitant & $63 / 428$ & 448 & 48 & Cs, Lzd & $\operatorname{Mfx}(400)$ & $438(5)$ & $\begin{array}{l}\text { Lzd (optic } \\
\text { neuritis) }\end{array}$ & No & 23 & Cure \\
\hline 2 & 29 & $\begin{array}{c}\mathrm{S}-\mathrm{C}+ \\
\mathrm{Cav}+\mathrm{Bil}-\end{array}$ & $\begin{array}{c}\text { Z, E, S, Ofx, Mfx, } \\
\text { Km, Eto, PAS, Cs, } \\
\text { Bdq }\end{array}$ & Concomitant & $113 / 636$ & 749 & 180 & PAS, Am, Lzd & $\operatorname{Mfx}(800), \mathrm{Cfz}$ & $459(21)$ & $\begin{array}{l}\text { PAS (vomiting), } \\
\text { Am (hearing loss) }\end{array}$ & No & 55 & Cure \\
\hline $3^{\S}$ & 20 & $\begin{array}{c}\mathrm{S}+\mathrm{C}+ \\
\mathrm{Cav}+\mathrm{Bil}+\end{array}$ & $\begin{array}{c}\text { Z, S, Ofx, Mfx, Am, } \\
\text { Km, Cm, Eto, Cs }\end{array}$ & Concomitant & $839 / 705$ & 839 & 705 & $\begin{array}{l}\text { E, PAS, Lzd, } \\
\text { Ipm, Amx/Clv }\end{array}$ & & $421(5)$ & & $\begin{array}{c}\text { Yes } \\
\text { (lobectomy) }\end{array}$ & 80 & Cure \\
\hline 4 & 32 & $\begin{array}{c}\mathrm{S}+\mathrm{C}+ \\
\mathrm{Cav}+\mathrm{Bil}+\end{array}$ & $\begin{array}{l}\text { Z, E, S, Ofx, Mfx, } \\
\text { Eto, PAS, Cs }\end{array}$ & Concomitant & $732 / 142$ & 732 & 147 & $\begin{array}{l}\text { Am, Lzd, Ipm, } \\
\text { Amx/Clv }\end{array}$ & $\mathrm{Cfz}$ & $489(13)$ & $\begin{array}{l}\text { DIm ladrenal } \\
\text { insufficiency) }\end{array}$ & $\begin{array}{c}\text { Yes } \\
\text { (lobectomy) }\end{array}$ & 89 & Cure \\
\hline 5 & 46 & $\begin{array}{c}\mathrm{S}+\mathrm{C}+ \\
\mathrm{Cav}+\mathrm{Bil}-\end{array}$ & $\begin{array}{l}\text { Z, E, S, Ofx, Mfx, } \\
\text { Km, Eto, Cs, }\end{array}$ & Concomitant & $355 / 324$ & 725 & 203 & Am, Cs, PAS & Mfx(800), Cfz & $>500(8)^{+}$ & $\begin{array}{l}\text { Mfx(800) (QT } \\
\text { prolongation), Am } \\
\text { (hearing loss) }\end{array}$ & No & 73 & Cure \\
\hline 6 & 30 & $\begin{array}{c}\mathrm{S}+\mathrm{C}+ \\
\mathrm{Cav}+\mathrm{Bil}+\end{array}$ & $\begin{array}{c}\text { Z, E, S, Ofx, Mfx, } \\
\text { Eto, Lzd }\end{array}$ & Concomitant & $735 / 700$ & 735 & 700 & $\begin{array}{l}\text { Am, Cs, PAS, } \\
\text { Ipm, Amx/Clv }\end{array}$ & $\mathrm{Cfz}$ & $>500$ (5) & $\begin{array}{c}\mathrm{Cfz} \text { (QT } \\
\text { prolongation) }\end{array}$ & No & 87 & Cure \\
\hline 7 & 33 & $\begin{array}{c}\mathrm{S}+\mathrm{C}- \\
\mathrm{Cav}+\mathrm{Bil}+\end{array}$ & $\begin{array}{c}\text { Z, E, S, Ofx, Mfx, } \\
\text { Am, Km, Cm, Eto, } \\
\text { Cs, Bdq }\end{array}$ & $\begin{array}{l}\text { Sequential } \\
\text { (20) }\end{array}$ & $56 / 286$ & 286 & 160 & $\begin{array}{l}\text { PAS, Lzd, Ipm, } \\
\text { Amx/Clv }\end{array}$ & $\mathrm{Cfz}$ & $472(1)$ & & No & $\mathrm{Na}$ & LTFU \\
\hline 8 & 36 & $\begin{array}{c}\mathrm{S}+\mathrm{C}- \\
\mathrm{Cav}+\mathrm{Bil}+\end{array}$ & $\begin{array}{c}\text { Z, E, S, Ofx, Mfx, } \\
\text { Km, Cm, Eto, Cs, } \\
\text { Bdq }\end{array}$ & $\begin{array}{l}\text { Sequential } \\
\text { (71) }\end{array}$ & $529 / 741$ & 741 & 109 & $\begin{array}{c}\text { Z, Am, Eto, Ipm, } \\
\text { Amx/Clv }\end{array}$ & & $481(1)$ & & $\begin{array}{c}\text { Yes } \\
\text { (lobectomy) }\end{array}$ & $\mathrm{Na}$ & Cure \\
\hline 9 & 40 & $\begin{array}{c}\mathrm{S}+\mathrm{C}+ \\
\mathrm{Cav}+\mathrm{Bil}+\end{array}$ & $\begin{array}{c}\text { Z, E, S, Ofx, Mfx, } \\
\text { Am, Km, Cm, Eto, } \\
\text { Bdq }\end{array}$ & $\begin{array}{l}\text { Sequential } \\
\text { (1) }\end{array}$ & $67 / 698$ & 744 & 180 & $\begin{array}{c}\mathrm{Cm}, \mathrm{Cs}, \mathrm{PAS}, \\
\mathrm{Lzd}\end{array}$ & Mfx(800), Cfz & $447(11)$ & $\begin{array}{l}\text { Lzd (peripheral } \\
\text { neuropathy) }\end{array}$ & No & 39 & Cure \\
\hline 10 & 25 & $\begin{array}{c}\mathrm{S}+\mathrm{C}+ \\
\mathrm{Cav}+\mathrm{Bil}+\end{array}$ & $\begin{array}{c}\text { Z, E, S, Ofx, Mfx, } \\
\text { Eto }\end{array}$ & $\begin{array}{l}\text { Sequential } \\
\text { (19) }\end{array}$ & $426 / 286$ & 731 & 161 & $\begin{array}{c}\text { Am, PAS, Cs, } \\
\text { Lzd }\end{array}$ & $\operatorname{Mfx}(400), \mathrm{Cfz}$ & 455 (13) & $\begin{array}{l}\text { Lzd (peripheral } \\
\text { neuropathy), Bdq } \\
\text { (oligoarthritis/ } \\
\text { osteonecrosis) }\end{array}$ & No & 88 & Cure \\
\hline
\end{tabular}

\#: for sequential treatment, time in days from bedaquiline discontinuation to delamanid start is shown in brackets; " exposure was calculated as the sum of concomitant treatment and for sequential treatment of bedaquiline and delamanid, time until the end of the washout period (180 days for bedaquiline); ${ }^{+}$: event occurred before the exposure to the bedaquiline/ delamanid combination; ${ }^{\S}$ : preliminary data of patient 3 have been previously described elsewhere [4]. TB: tuberculosis; QTcF: QT interval corrected according to the Fridericia formula; S+: sputum smear positive at treatment start; $\mathrm{S}_{-}$: sputum smear negative at treatment start; $\mathrm{C}_{+}$: sputum culture positive at treatment start; $\mathrm{C}_{-}$: sputum culture negative at treatment start; Cav+: presence of at least one lung cavity at treatment start; Bil+: bilateral lung involvement at treatment start; Bil-: unilateral lung involvement at treatment start; LTFU: lost to follow-up; R: rifampicin; H: isoniazid; Bdq: bedaquiline; Dlm: delamanid; Z: pyrazinamide; E: ethambutol; S: streptomycin; Ofx: ofloxacin; Mfx(400): moxifloxacin (400 mg.day ${ }^{-1}$ ); Mfx(800): moxifloxacin (800 mg.day ${ }^{-1}$ ); Am: amikacin; $\mathrm{Km}$ : kanamycin; $\mathrm{Cm}$ : capreomycin; Eto: ethionamide; Cs: cycloserine; PAS: para-aminosalicylic acid; Lzd: linezolid; Cfz: clofazimine; Ipm: imipenem; Amx/Clv: amoxicillin/clavulanic acid; na: patient was sputum culture negative at treatment start. 
Overall, 10 patients were included in the study (table 1). All patients were male; nine were previously treated for TB. The median age was 32 years (interquartile range (IQR) 28-41 years). All patients had a phenotypic drug susceptibility test showing resistance to rifampicin and isoniazid at baseline, plus additional resistance to a median of eight drugs (IQR 6-9). Six fulfilled the definition of XDR-TB, and four had fluoroquinolone-resistant MDR-TB. Resistance to bedaquiline was detected in four cases, as previously reported [10]. In three cases (patients 2, 7 and 9), bedaquiline resistance was found in the baseline specimen and led to replacement of the drug with delamanid. Patient 8 developed bedaquiline resistance on treatment, as reported elsewhere [8], and started a new delamanid-containing regimen. All patients had cavitary pulmonary TB, eight with bilateral lung involvement. Nine patients had smear-positive sputum at baseline. None had extrapulmonary TB. Four had PCR-positive HCV infection and one had chronic HBV hepatitis. No patient had HIV infection, or diabetes. Overall, six patients received concomitant bedaquilinedelamanid treatment, and four were treated initially with bedaquiline and then switched to delamanid without (or with incomplete) washout. Six patients received prolonged ( $>24$ weeks) treatment with bedaquiline, and nine with delamanid: respective median treatment durations were 391 (IQR 66-733) and 532 (IQR 286-701) days. Median exposure to the combination of bedaquiline and delamanid was 171 (IQR 138-327) days, with two patients receiving concomitantly the two drugs for the full treatment course. The most commonly co-administered drugs were clofazimine (70\%) and linezolid (60\%). Notably, four patients received one QT-prolonging drug, namely clofazimine or moxifloxacin, and four received both. Three patients underwent lung surgery (lobectomy) after a median of 125 (range 114-328) days from treatment start; all of them had converted their sputum cultures prior to the surgery date. All patients experienced at least one adverse event, and four patients had a serious adverse event (table 1). No $>60 \mathrm{~ms}$ QTcF increase occurred during treatment. QTcF $>500 \mathrm{~ms}$ values were reported in two patients. One was receiving a regimen including bedaquiline, delamanid and clofazimine; his QTcF interval reverted to normal after the discontinuation of clofazimine. The other was on treatment with a regimen which included bedaquiline and moxifloxacin at $800 \mathrm{mg} \cdot \mathrm{day}^{-1}$; after the discontinuation of moxifloxacin, the QTcF interval decreased below $500 \mathrm{~ms}$, allowing the introduction of delamanid. Overall, no arrhythmia or other cardiac event was recorded. Delamanid was discontinued in a patient who developed adrenal insufficiency, and bedaquiline was discontinued in a patient experiencing oligoarthritis and osteonecrosis. Out of eight patients with a positive sputum culture at treatment initiation, all achieved sputum culture conversion after 3 months of treatment (median time to culture conversion 77 (IQR 43-88) days). Overall, nine patients were cured and one was lost to follow-up after 8 months of treatment; at the last visit, he had culture-negative sputum.

We report the use of bedaquiline and delamanid in 10 MDR-TB patients. Prolonged periods of exposure to these two drugs, including use beyond 24 weeks, appeared to be rather well tolerated. QTcF $>500 \mathrm{~ms}$ was recorded in two cases of co-administration of multiple QT-prolonging drugs; however, the overall cardiac safety was good in these patients, confirming recent studies [11, 12]. Regular ECG monitoring allowed timely identification and management QT prolongation above $500 \mathrm{~ms}$, which may have contributed in preventing cardiac events. The adverse events that led to the discontinuation of bedaquiline (patient 10) and delamanid (patient 4) had not been described before in association with these drugs, and therefore causality assessment was uncertain. Successful treatment outcomes were observed for nine of the 10 patients, despite advanced resistance patterns, including fluoroquinolone resistance in all cases. Overall, these results suggest that, in cases where scarce therapeutic options are available, these drugs could be used concomitantly, rather than sequentially with washout, to prevent the selection of drug resistance [13]. This study has some limitations, including the small sample size, retrospective data collection, and unavailability of systematic monitoring of blood levels of bedaquiline and delamanid.

In conclusion, our results show good tolerance of exposure to the combination of bedaquiline and delamanid for the treatment of MDR/XDR-TB. Ongoing clinical trials (DELIBERATE (NCT02583048), and endTB (NCT02754765)) will provide high-quality evidence on the combination. Meanwhile, these findings should encourage considering this treatment option for patients with limited therapeutic alternatives $[14,15]$.

Lorenzo Guglielmetti $\oplus^{1,2,3}$, Linda Barkane $e^{4,5}$, Damien Le D $\hat{u}^{3}$, Dhiba Marigot-Outtandy ${ }^{3,6}$, Jérôme Robert ${ }^{1,2}$, Nicolas Veziris ${ }^{1,2,7}$, Yazdan Yazdanpanah ${ }^{8}$, Liga Kuksa ${ }^{4,5}$, Eric Caumes ${ }^{9}$ and Mathilde Fréchet-Jachym ${ }^{3}$

${ }^{1}$ APHP, Centre National de Référence des Mycobactéries et de la Résistance des Mycobactéries aux Antituberculeux (CNR-MyRMA), Bactériologie-Hygiène, Hôpitaux Universitaires Pitié Salpêtrière-Charles Foix, Paris, France. ${ }^{2}$ Sorbonne Université, Université Pierre et Marie Curie 06, Unité 1135, Team E13 (Bactériologie), CR7 INSERM, Centre d'Immunologie et des Maladies Infectieuses, Paris, France. ${ }^{3}$ Sanatorium, Centre Hospitalier de Bligny, Briis-sous-Forges, France. ${ }^{4}$ Riga East University Hospital TB and Lung Disease Centre, Riga, Latvia. ${ }^{5}$ Riga Stradins University, Riga, Latvia. ${ }^{6}$ APHP, Service de Médecine Aigue Spécialisée, Hôpital Raymond Poincaré, Garches, France. ${ }^{7}$ APHP, Hôpitaux Universitaires de l'Est Parisien, Paris, France. ${ }^{8}$ APHP, Service des Maladies Infectieuses et Tropicales, Hôpital Bichat Claude Bernard, Paris, France. ${ }^{9}$ APHP, Service des Maladies Infectieuses et Tropicales, Hôpitaux Universitaires Pitié Salpêtrière-Charles Foix, Paris, France. 
Correspondence: Lorenzo Guglielmetti, Laboratoire de Bactériologie-Hygiène, Faculté de Médecine Pierre et Marie Curie (UPMC Paris 6), 91 Boulevard de l'hôpital, 75634 Paris Cedex 13, France. E-mail: lorenzo.guglielmetti@gmail.com

Received: Dec 082017 | Accepted after revision: Jan 072018

Author contributions: L. Guglielmetti made a substantial contribution to the conception and design of the work, to the acquisition, analysis and interpretation of data for the work, performed statistical analysis, wrote the manuscript, critically revised the manuscript for important intellectual content, and gave final approval of the current version to be published. L. Barkane, D. Le Du and M. Fréchet-Jachym made a substantial contribution to the conception and design of the work, to the acquisition, analysis and interpretation of data for the work, wrote the manuscript, critically revised the manuscript for important intellectual content, and gave final approval of the current version to be published. All other authors made a contribution to the acquisition of the data for the work, critically revised the manuscript for important intellectual content, and gave final approval of the current version to be published. All authors agree to be accountable for all aspects of the work in ensuring that questions related to the accuracy or integrity of any part of the work are appropriately investigated and resolved.

Conflict of interest: L. Guglielmetti belonged to a research team that received research grants from Janssen Pharmaceuticals, outside the submitted work. L. Barkane was sub-investigator in the delamanid trial 213 and presented the Latvian experience with delamanid in the ERS congress 2016. J. Robert belonged to a research team that received research grants from Janssen Pharmaceuticals, outside the submitted work. N. Veziris received financial support for attending the 48th Union World Conference on Lung Health from Otsuka, outside the submitted work. Y. Yazdanpanah has been a board member receiving consultancy fees from ABBVIE, BMS, Gilead, MSD, J\&J, Pfizer and ViiV Healthcare until November 2016. L. Kuksa was an investigator in the delamanid trials 204, 208 and 213 and presented Latvia experience with the compound at the launching conference in Korea.

Acknowledgements: The authors acknowledge Camille Diallo, Bénédicte Lemaire and Dominique Szmigiel-Perret for their help in conducting the study.

\section{References}

1 Bastos ML, Lan Z, Menzies D. An updated systematic review and meta-analysis for treatment of multidrug-resistant tuberculosis. Eur Respir J 2017; 49: 1600803.

2 Diacon AH, Pym A, Grobusch MP, et al. Multidrug-resistant tuberculosis and culture conversion with bedaquiline. N Engl J Med 2014; 371: 723-732.

3 Skripconoka V, Danilovits $\mathrm{M}$, Pehme L, et al. Delamanid improves outcomes and reduces mortality in multidrug-resistant tuberculosis. Eur Respir J 2012; 41: 1393-1400.

4 Lachatre M, Rioux C, Le Du D, et al. Bedaquiline plus delamanid for XDR tuberculosis. Lancet Infect Dis 2016; 16: 294.

5 Tadolini M, Lingtsang RD, Tiberi S, et al. First case of extensively drug-resistant tuberculosis treated with both delamanid and bedaquiline. Eur Respir J 2016; 48: 935-938.

6 Maryandyshev A, Pontali E, Tiberi S, et al. Bedaquiline and delamanid combination treatment of 5 patients with pulmonary extensively drug-resistant tuberculosis. Emerg Infect Dis 2017; 23: 1718-1721.

7 World Health Organization. Companion handbook to the WHO guidelines for the programmatic management of drug-resistant tuberculosis. Geneva, WHO, 2014.

8 Guglielmetti L, Jaspard M, Le Du D, et al. Long-term outcome and safety of prolonged bedaquiline treatment for multidrug-resistant tuberculosis. Eur Respir J 2017; 49: 1601799.

9 National Cancer Institute. Common Terminology Criteria for Adverse Events, version 4.0. http://evs.nci.nih.gov/ ftp1/CTCAE Date last accessed: September 15, 2017.

10 Veziris N, Bernard C, Guglielmetti L, et al. Rapid emergence of Mycobacterium tuberculosis bedaquiline resistance: lessons to avoid repeating past errors. Eur Respir J 2017; 49: 1601719.

11 Yoon H-Y, Jo K-W, Nam GB, et al. Clinical significance of QT-prolonging drug use in patients with MDR-TB or NTM disease. Int I Tuberc Lung Dis 2017; 21: 996-1001.

12 Pontali E, Sotgiu G, Tiberi S, et al. Cardiac safety of bedaquiline: a systematic and critical analysis of the evidence. Eur Respir J 2017; 50: 1701462.

13 Bloemberg GV, Gagneux S, Boettger EC. Acquired resistance to bedaquiline and delamanid in therapy for tuberculosis. N Engl J Med 2015; 373: 1986-1988.

14 Guglielmetti L, Le Du D, Jachym $\mathrm{M}$, et al. Preventing acquired resistance to bedaquiline and delamanid in multidrug-resistant tuberculosis treatment requires optimal management. Am J Respir Crit Care Med 2016; 194: $1170-1171$.

15 Guglielmetti L. Bedaquiline for the treatment of multidrug-resistant tuberculosis: another missed opportunity? Eur Respir J 2017; 49: 1700738. 